\title{
Heavy-light meson in anisotropic lattice QCD*
}

\author{
H. Matsufuru ${ }^{\mathrm{a}}$, J. Harada ${ }^{\mathrm{b}}$, T. Onogi ${ }^{\mathrm{a}}$ and A. Sugita ${ }^{\mathrm{a}}$ \\ ${ }^{a}$ Yukawa Institute for Theoretical Physics, Kyoto University, Kyoto 606-8502, Japan \\ ${ }^{\mathrm{b}}$ Department of Physics, Hiroshima University, Higashi-hiroshima 739-8526, Japan
}

We examine whether the $O(a)$ improved quark action on anisotropic lattices can be used as a framework for the heavy quark, which enables precision computation of matrix elements of heavy-light mesons. To this end, it is crucial to verify that a mass independent and nonperturbative tuning of the parameters is possible. As a first step, we observe the dispersion relation of heavy-light mesons on a quenched lattice using the action which is nonperturbatively tuned only for the leading terms. On a lattice with the spatial cutoff $a_{\sigma}^{-1} \simeq 1.6 \mathrm{GeV}$ and the anisotropy $\xi=4$, the relativity relation holds within $2 \%$ accuracy in the quark mass region $a_{\sigma} m_{Q} \leq 1.2$ with the bare anisotropy parameter tuned for the massless quark. We also apply the action to a calculation of heavy-light decay constants in the charm quark mass region.

\section{Introduction}

Recent experimental progress at $B$ factories suggests that precise computation of hadronic matrix elements in lattice QCD is a key to the search for signals of new physics in flavor physics.

However, the two major formalisms for the heavy quark, i.e. HQET approaches or the relativistic approaches to describe the heavy quarks on the lattice still suffer from perturbative and/or discretization errors, which are typically $\sim 10 \%$. We therefore need yet another formalism of the heavy quark for the precise and systematic computation of matrix elements.

Ideally, in such a formalism, one should be able to (i) take the continuum limit, (ii) compute the parameters in the action and the operators nonperturbatively, (iii) and compute matrix elements with a modest computational cost.

As a candidate of framework which fulfills these conditions, we investigate the anisotropic lattice with the temporal lattice spacing $a_{\tau}$ finer than the spatial one $a_{\sigma}$ [1],2,3]. Anisotropic lattice framework evidently satisfies above conditions (i) and (iii). Our expectation is that on anisotropic lattices the mass dependence of the parameters becomes so mild that one can adopt coefficients determined nonperturbatively at massless limit. Whether these promises will be practically satisfied should be examined numerically.

\footnotetext{
*Talk presented by H. Matsufuru
}

As a first step toward this goal, we study the action which is nonperturbatively tuned only for the leading term, while $O(a)$ improvement terms are tuned at the tadpole tree level. We measure the heavy-light meson dispersion relation with the anisotropy parameter tuned at the massless limit. We can then study the breaking of relativity condition as a function of the heavy quark mass, with which we can test if the small mass dependence of the parameter really holds.

\section{Anisotropic lattice quark action}

Our heavy quark formalism basically follows the Fermilab approach [n but is formulated on the anisotropic lattice [1, 5].

The quark action is represented as

$$
\begin{aligned}
& S_{F}= \sum_{x, y} \bar{\psi}(x) K(x, y) \psi(y) \\
& K(x, y)=\delta_{x, y}-\kappa_{\tau}\left[\left(1-\gamma_{4}\right) U_{4}(x) \delta_{x+\hat{4}, y}\right. \\
&\left.+\left(1+\gamma_{4}\right) U_{4}^{\dagger}(x-\hat{4}) \delta_{x-\hat{4}, y}\right] \\
&-\kappa_{\sigma} \sum_{i}\left[\left(r-\gamma_{i}\right) U_{i}(x) \delta_{x+\hat{i}, y}\right. \\
&\left.+\left(r+\gamma_{i}\right) U_{i}^{\dagger}(x-\hat{i}) \delta_{x-\hat{i}, y}\right] \\
&-\kappa_{\sigma} c_{E} \sum_{i} \sigma_{4 i} F_{4 i}(x) \delta_{x, y} \\
&-r \kappa_{\sigma} c_{B} \sum_{i>j} \sigma_{i j} F_{i j}(x) \delta_{x, y}
\end{aligned}
$$

where $\kappa_{\sigma}$ and $\kappa_{\tau}$ are the spatial and temporal 
hopping parameters, $r$ is the Wilson parameter and $c_{E}$ and $c_{B}$ are the clover coefficients.

In principle for a given $\kappa_{\sigma}$, the four parameters $\kappa_{\sigma} / \kappa_{\tau}, r, c_{E}$ and $c_{B}$ should be tuned so that Lorentz symmetry holds up to discretization errors of $O\left(a^{2}\right)$.

In this work, we set the spatial Wilson parameter as $r=1 / \xi$ and the clover coefficients as the tadpole-improved tree-level values as $c_{E}=$ $1 / u_{\sigma} u_{\tau}^{2}, c_{B}=1 / u_{\sigma}^{3}$ and perform a nonperturbative calibration only for $\gamma_{F}$. The tadpole improvement [6] is achieved by rescaling the link variables as $U_{i}(x) \rightarrow U_{i}(x) / u_{\sigma}$ and $U_{4}(x) \rightarrow U_{4}(x) / u_{\tau}$, with the mean-field values of the spatial and temporal link variables, $u_{\sigma}$ and $u_{\tau}$, respectively. This is equivalent to redefining the hopping parameters with the tadpole-improved ones (with tilde) through $\kappa_{\sigma}=\tilde{\kappa}_{\sigma} / u_{\sigma}$ and $\kappa_{\tau}=\tilde{\kappa}_{\tau} / u_{\tau}$. We define the anisotropy parameter $\gamma_{F}$ as $\gamma_{F} \equiv \tilde{\kappa}_{\tau} / \tilde{\kappa}_{\sigma}$.

For convenience, we also introduce $\kappa$ as

$\frac{1}{\kappa} \equiv \frac{1}{\tilde{\kappa}_{\sigma}}-2\left(\gamma_{F}+3 r-4\right)=2\left(m_{0} \gamma_{F}+4\right)$,

where $m_{0}$ is the bare quark mass in temporal lattice units. $\gamma_{F}$ is the bare anisotropy parameter, which should be tuned so that the physical fermionic anisotropy $\xi_{F}$ retains the same value as that of the gauge field, $\xi_{G}$. For the quenched case, as for the present work, one can first calibrate the anisotropy parameter of gauge field separately from the quark field, and then tune $\gamma_{F}$ so that $\xi_{F}=\xi_{G}=\xi$ holds.

The calibration of bare anisotropy $\gamma_{F}$ was performed in Ref. [2] using the dispersion relation of mesons for quark masses less than the charm quark mass region, on quenched lattices with $\xi=4$ for three different lattice spacings. The quark mass dependence is indeed mild and well represented by a linear form in $m_{q}^{2}$.

\section{Relativity condition}

We next study whether the relativistic dispersion relation holds for the heavy-light mesons with $\gamma_{F}$ tuned for the massless quark [3]. In general, the lattice dispersion relation for arbitrary values of $\gamma_{F}$ is described as

$E^{2}(\boldsymbol{p})=m^{2}+\boldsymbol{p}^{2} / \xi_{F}^{2}+O\left(p^{4}\right)$,

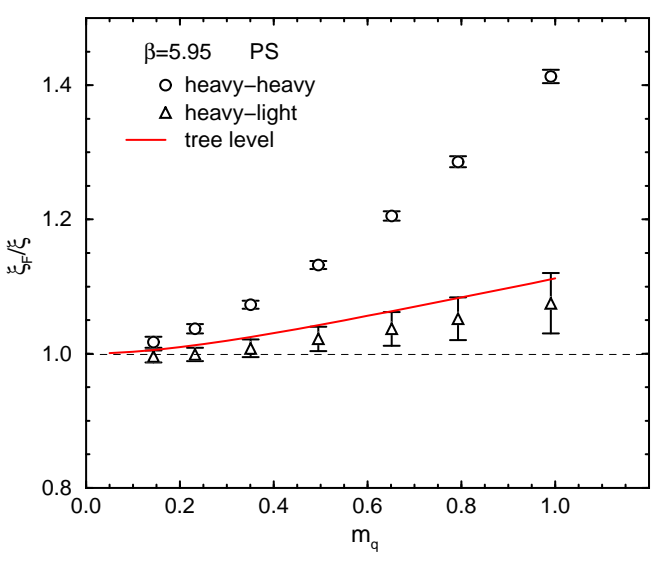

Figure 1. The heavy quark mass dependence of the anisotropy $\xi_{F}$ for heavy-light and heavyheavy mesons at $\beta=5.95$.

where the energy $E$ and the rest mass $m$ are in temporal lattice units while the momentum $\boldsymbol{p}$ is in spatial units. The parameter $\xi_{F}$ in this equation defines the anisotropy of the quark fields, whose difference with the gauge field anisotropy $\xi$ probes the breaking of relativity.

Numerical simulations are performed on a quenched lattice of the size $16^{3} \times 128$ generated with the Wilson plaquette action at $\beta=6.10$ for $\xi=4$, which is realized by taking the bare anisotropy of gauge field $\gamma_{G}$ using the numerical formula determined by Klassen in one percent accuracy [7]. The lattice cutoff defined through the hadronic radius $r_{0}$ is $a_{\sigma}^{-1}=1.623(9) \mathrm{GeV}$. Other details of parameters such as the mean-field values are found in Refs. [2,3].

The result for the pseudoscalar meson is displayed in Fig. 11. Similar result is obtained for the vector channel. The measured value $\xi_{F}$ for the heavy-light meson coincides with $\xi$ in the quark mass region $m_{q} \leq 0.3$ (in temporal lattice units) within $2 \%$ accuracy. (This accuracy is just a guide for argument of uncertainty in this paper.) Therefore, for the range of quark mass $m_{q} \leq 0.3$ which covers the charm quark mass with present lattice cutoff, our quark action with the bare anisotropy tuned for massless quark can successfully describe the heavy-light meson with controlled systematic uncertainty. We also show 
the $\xi_{F}$ of the heavy quarkonium. In this case, $\xi_{F}$ rapidly increases because of large momentum transfer inside heavy quarkonium, $p \simeq \alpha m_{Q}$, in contrast to the case of heavy-light systems, where $p \simeq \Lambda_{Q C D}$.

\section{Heavy-light decay constant}

The result in the previous section tells us that the framework can be applied to charmed heavy-light meson systems. As a first application, we compute the heavy-light decay constants. Numerical simulations are performed on two quenched lattices at $\beta=5.95$ and 6.10 with the renormalized anisotropy $\xi=4$. The former is the same as in previous section, and the latter has spatial cutoff $a_{\sigma}^{-1}=2.030(13) \mathrm{GeV}$. For the light quark, we use three values of the hopping parameter $\kappa$ which cover the range of quark masses from $m_{s}$ to $1.5 m_{s}$. The chiral limit is taken by a linear extrapolation. The matching to the continuum theory is at the tadpole improved tree level. The result at $\beta=6.0$ is displayed in Fig. 2. In this analysis, the physical quark masses are defined through the lattice cutoff defined with the $K^{*}$ meson mass. We find that the behavior of $f \sqrt{m}$ is consistent with the results on isotropic lattices. Since the currents are matched only at the tadpole tree level and the $O(a)$ improvement terms are tuned also only at the tadpole tree level, the present calculation contains rather large renormalization uncertainty as well as the cutoff dependence. In order to suppress these errors we compute the ratio of decay constants. Our preliminary results are

$$
\begin{aligned}
& f_{D} / f_{\pi}=1.566(43) \text { at } \beta=5.95, \\
& 1.515(43) \text { at } \beta=6.10 \text {, } \\
& f_{D_{s}} / f_{D}=1.140(14) \text { at } \beta=5.95 \text {, } \\
& 1.142(14) \text { at } \beta=6.10 \text {, }
\end{aligned}
$$

which are consistent with previous works on isotropic lattices [8]. A remarkable point is that the lattice spacing dependence of the ratio is small. In fact, individual decay constants could suffer from both the mass independent and mass dependent errors, but the former can largely cancel in the ratio. Therefore, the stability of the

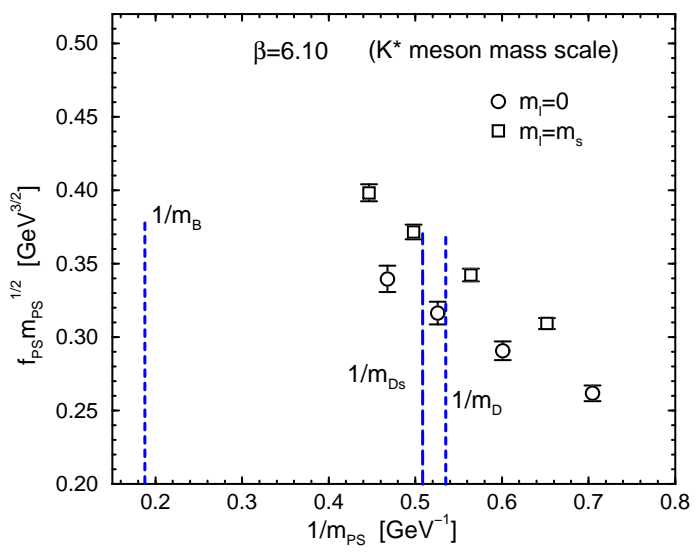

Figure 2. Heavy-light meson decay constant in the pseudoscalar channel at $\beta=6.10$.

ratio may be an indication that the mass dependent errors are suppressed in our formalism on anisotropic lattices as expected.

In conclusion, the results of numerical simulations in quenched lattices are encouraging for further development in this direction. Further improvements, such as nonperturbative tuning of the clover coefficients, are necessary for achieving the desired accuracy.

The simulations were done on a NEC SX-5 at RCNP, Osaka University and a Hitachi SR8000 at KEK.

\section{REFERENCES}

1. J. Harada et al., Phys. Rev. D 64 (2001) 074501.

2. H. Matsufuru, T. Onogi and T. Umeda, Phys. Rev. D 64 (2001) 114503.

3. J. Harada, H. Matsufuru, T. Onogi and A. Sugita, Phys. Rev. D 66 (2002) 014509.

4. A. X. El-Khadra, A. S. Kronfeld and P. B. Mackenzie, Phys. Rev. D 55 (1997) 3933.

5. T. Umeda et al., Int. J. Mod. Phys. A 16 (2001) 2215.

6. G. P. Lepage and P. B. Mackenzie, Phys. Rev. D 48 (1993) 2250.

7. T. R. Klassen, Nucl. Phys. B 533 (1998) 557.

8. S. Ryan, Nucl. Phys. B (Proc. Suppl.) 106 (1994) 839. 